\title{
Performance of a Direct Electron Detector for the Application of Electron Energy- Loss Spectroscopy
}

\author{
James L. Hart ${ }^{1}$, Andrew C. Lang ${ }^{1}$, Colin Trevor $^{2}$, Ray Twesten ${ }^{2}$, Mitra L. Taheri ${ }^{1}$ \\ 1. Department of Materials Science and Engineering, Drexel University, Philadelphia PA, USA \\ 2. Analytic Projects R\&D, Gatan, Pleasanton CA, USA
}

Transmission electron microscopes (TEMs) conventionally employ indirect detection cameras (IDC) for electron imaging. Such IDCs consist of a scintillator and a digital imaging device with a lens or fiber optic network coupling photons from the scintillator to the camera. Alternatively direct detection cameras (DDC) directly image electrons. Compared to IDCs, DDCs offer an improved point spread function (PSF), lower read-out noise, and potential for higher frame rates [1,2]. DDCs have been successfully utilized by the cryo-TEM community [3] and, more recently, for in-situ TEM applications [4]. Here we evaluate a DDC for the application of electron energy-loss spectroscopy (EELS). We compared the performance of a Gatan K2 Summit (DDC) with a Gatan US1000FTXP (IDC). Both detectors were mounted to a Gatan GIF Quantum energy filter. Our results show that the narrow PSF of the DDC improves measured resolution given a fixed beam energy spread and spectrometer dispersion. Additionally, the low read-out noise of the DDC increases spectrum signal to noise (SNR) for short acquisition times. These results indicate DDCs will enable efficient acquisition of low-noise spectra for applications ranging from in-situ EELS to low-dose chemical mapping.

Figure 1 shows a zero loss peak (ZLP) captured with each detector. In both cases the spectrometer was set to $1 \mathrm{eV} /$ channel dispersion. The DDC was mounted on a FEI T20 ( $\left.\mathrm{LaB}_{6}\right)$, which has an energy spread full width at half maximum $(\mathrm{FWHM}) \approx 1 \mathrm{eV}$. The detected ZLP has a FWHM of $1 \mathrm{eV}$, demonstrating that the DDC is able to accurately detect the ZLP without significant signal spreading, a result of the DDC's narrow PSF. The IDC, mounted on a FEI TF20 (Schottkey) with a FWHM $\approx 0.8 \mathrm{eV}$, recorded a ZLP with a FWHM of $3 \mathrm{eV}$. The comparatively broad IDC PSF prevents accurate detection of the ZLP at this low dispersion.

A $\mathrm{SrTiO}_{3}$ sample was investigated with each detector. Figure 2 shows Ti L edge spectra acquired with three different acquisition times. All spectra acquired with the DDC display splitting of the $\mathrm{Ti}_{2,3}$ edges, however, spectra acquired with the IDC detector do not show splitting. Again, this result is attributed to the differing PSFs of the detectors. The narrow PSF of the DDC is able to resolved the Ti $\mathrm{L}_{2,3}$ edge splitting while to broader PSF of the IDC cannot resolve the splitting at this dispersion.

For each spectra shown in Figure 2, pre-edge SNR values were calculated (Table 1). A 50 eV pre-edge window was defined and SNR was calculated as $\mathrm{SNR}=N / \sigma$, where $N$ is the average number of counts and $\sigma$ is the signal standard deviation. For spectra acquired with 10.0 and 1.0 second acquisition times, the IDC provided higher SNR while the DDC provided higher SNR for 0.1 second acquisition time. This transition is due to the interplay between shot noise, read-out noise, detector PSF, and electron dosage. For short acquisition times, read-out noise contributes significantly to total spectrum noise. In this regime, the DDC provides higher SNR because of its low read-out noise. For longer acquisition times, read-out noise remains constant while shot noise increases, becoming the dominant noise component. Shot noise is inherent to the pre-detected signal, and is therefore convoluted by the detector PSF. In this limit, the IDC provides higher SNR owing to its broader PSF [5]. 
References:

[1] R. S. Ruskin, Z. Yu, and N. Grigorieff, J. Struct. Biol. 184, 385 (2013).

[2] D. Contarato, P. Denes, D. Doering, J. Joseph, and B. Krieger, Phys. Procedia 37, 1504 (2012).

[3] X. Li et al, Nat. Methods 10, 584 (2013).

[4] E. A. Stach et al, Microcopy Microanal. 19, 392 (2013).

[5] The authors acknowledge financial support from NSF MRI award \# 1429661
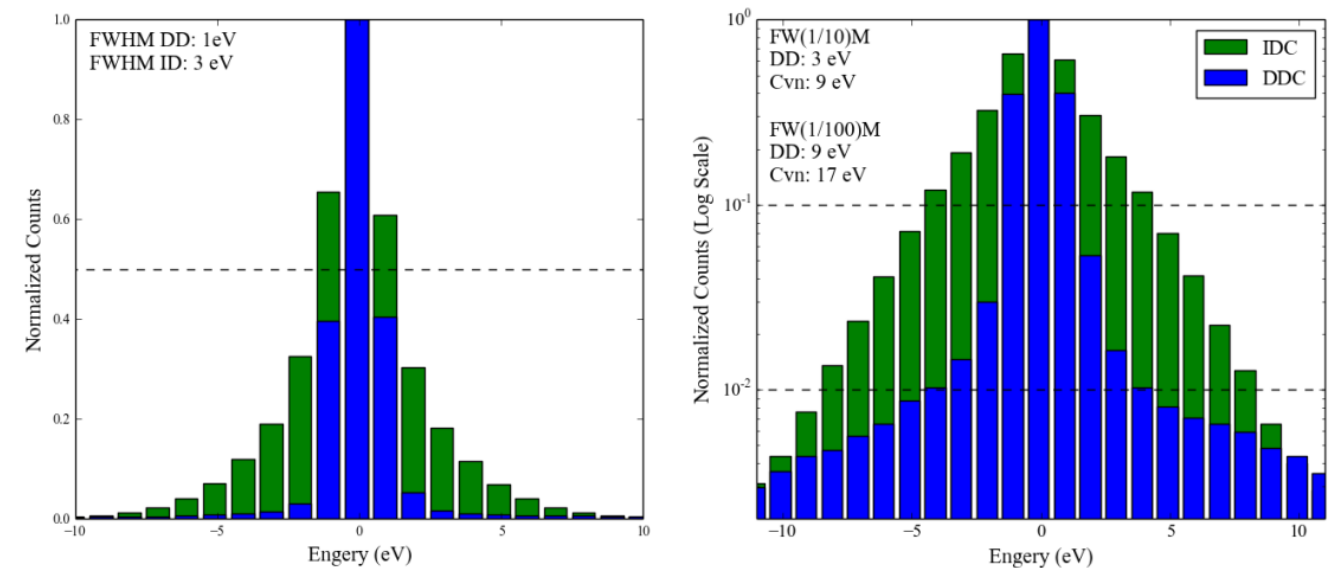

Figure 1. ZLPs captured with each detector, shown on a linear scale (left) and a log scale (right).
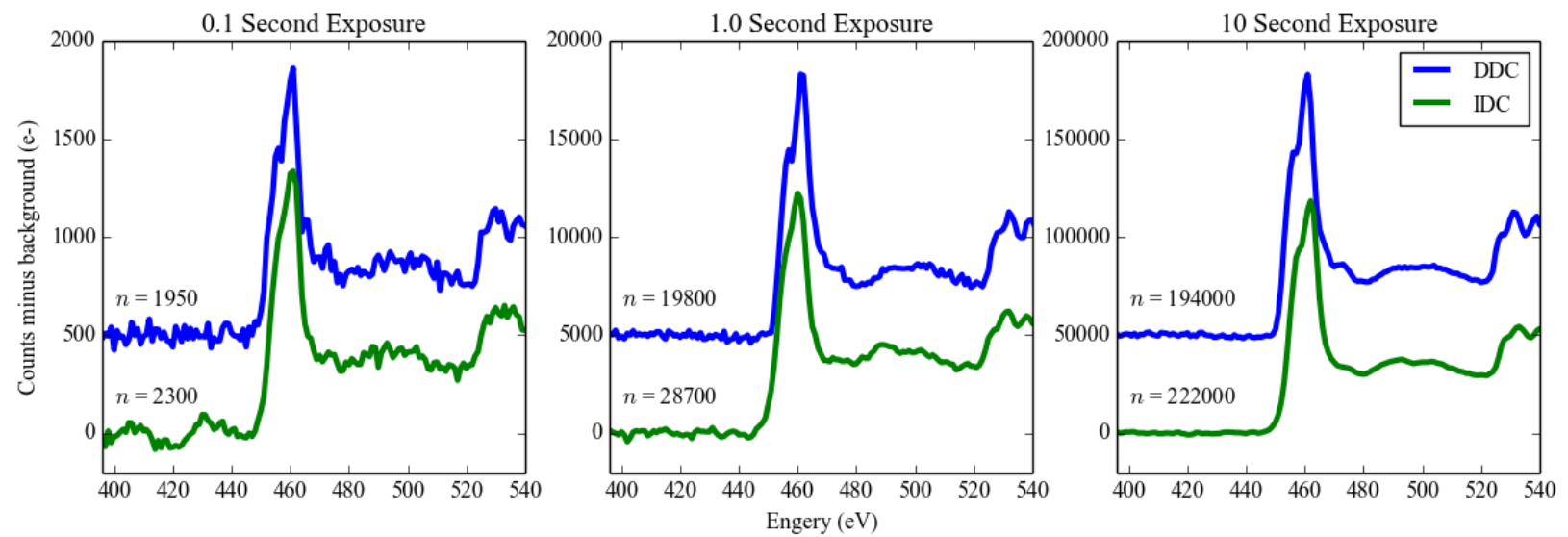

Figure 2. $\mathrm{Ti} \mathrm{L}_{2,3}$ spectra captured with each detector at three different acquisition times. Background subtraction was performed with a power-law fit. Average pre-edge counts are shown for each spectra.

Table 1: Comparison of the experimental and simulated SNR results for each detector. Higher SNR values are in bold. Our simulations account for electron dosage, shot noise, read-out noise, noise due to gain variation, and detector PSF profiles extracted from experimental ZLPs.

\begin{tabular}{|r|r|r|r|r|}
\hline \multicolumn{5}{|c|}{ SNR Results } \\
\hline $\begin{array}{r}\text { Acquisition } \\
\text { Time (s) }\end{array}$ & \multicolumn{2}{|c|}{ DDC } & \multicolumn{2}{c|}{ IDC } \\
\cline { 2 - 5 } & Exp & Sim & Exp & Sim \\
\hline 0.1 & $\mathbf{5 2}$ & $\mathbf{5 3}$ & 49 & 48 \\
\hline 1.0 & 148 & 175 & $\mathbf{2 1 2}$ & $\mathbf{3 0 5}$ \\
\hline 10 & 253 & 252 & $\mathbf{4 9 9}$ & $\mathbf{4 9 7}$ \\
\hline
\end{tabular}

